\title{
ARTICLE OPEN \\ Electric field tuning of the anomalous Hall effect at oxide interfaces
}

\author{
Sayantika Bhowal iD ${ }^{1}$ and Sashi Satpathy ${ }^{1}$
}

Anomalous Hall effect is the phenomenon where the transport properties of the spin-polarized electrons are governed by the spinorbit coupling that couples the orbital and spin degrees of freedom of the electron. Here we show that the anomalous Hall effect at a magnetic interface with strong spin-orbit coupling can be tuned with an external electric field. By altering the strength of the inversion symmetry breaking, the electric field changes the Rashba interaction, which in turn modifies the magnitude of the Berry curvature, the central quantity in determining the anomalous Hall conductivity. The effect is illustrated with a square lattice model, which yields a quadratic dependence of the anomalous Hall conductivity for small electric fields. Explicit density-functional calculations were performed for the recently grown iridate interface, viz., the $\left(\mathrm{SrlrO}_{3}\right)_{1} /\left(\mathrm{SrMnO}_{3}\right)_{1}(001)$ structure, both with and without an electric field, which show a strong electric field dependence. The effect may be potentially useful in spintronics applications.

npj Computational Materials (2019)5:61; https://doi.org/10.1038/s41524-019-0198-8

\section{INTRODUCTION}

The anomalous Hall effect (AHE) occurs in solids with broken timereversal symmetry, such as the ferromagnets, as a result of the spin-orbit coupling (SOC). Although the effect was noticed in the original work of Hall himself, ${ }^{1,2}$ the explanation of the phenomenon came from the seminal paper of Karplus and Luttinger, ${ }^{3}$ where they identified the anomalous contribution to arise from the SOC, which results in the left-right asymmetry in the scattering of the spin-polarized electrons. Currently, there is a considerable interest on the AHE from a technological point of view because of potential applications in spintronics such as for magnetic sensors and memory devices. ${ }^{4}$

The interface between $3 d$ anti-ferromagnetic insulator $\mathrm{SrMnO}_{3}$ $(\mathrm{SMO})^{5}$ and $5 d$ paramagnetic metal $\mathrm{SrlrO}_{3}(\mathrm{SIO})^{6-8}$ is one of the notable examples among several attempts ${ }^{9-13}$ to engineer the electronic and magnetic properties at the $3 d-5 d$ interfaces, where the strong coupling is achieved by the charge transfer from SIO to the SMO side, ${ }^{9,14}$ as sketched in Fig. 1. This results in electron doped SMO and hole doped SIO, which leads to an emergent ferromagnetism at the interface. The ferromagnetism at the interface in turn gives rise to the $\mathrm{AHE}$, which has been measured for the short-period superlattices of SIO/SMO. ${ }^{9}$

In this paper, we show that the AHE can be tuned by an external electric field by modifying the strength of the Rashba interaction. The idea that the Rashba interaction can modify the AHE is a reasonable expectation, since any kind of magnetic field would affect the Hall conductivity and the Rashba interaction is equivalent to a magnetic field, albeit $\mathrm{k}$ dependent. Here, we study the effect using general arguments as well as from densityfunctional calculations of the anomalous Hall conductivity (AHC) for a specific interface structure $(\mathrm{SIO})_{1} /(\mathrm{SMO})_{1}$. Similar interface structures have already been grown experimentally. ${ }^{9}$ Such a perovskite hetero-structure is a good candidate for the electric field control of the Rashba effect, ${ }^{15,16}$ providing an excellent platform for the manipulation of the AHE.

\section{RESULTS AND DISCUSSIONS}

To illustrate the effect of the electric field on $A H E$, consider the motion of electrons in a simplified tight-binding (TB) model of a ferromagnetic square lattice [Fig. 2a], relevant for the transition metal atoms at the interface. The Hamiltonian is

$$
\begin{aligned}
\mathcal{H}= & \mathcal{H}_{\text {kin }}+\mathcal{H}_{e x}+\mathcal{H}_{S O c}+\mathcal{H}_{E} \\
= & \sum_{i \mu \sigma, j \nu \sigma} t_{i j}^{\mu \nu} c_{i \mu \sigma}^{\dagger} c_{j \nu \sigma}-J_{e x} \sum_{i \mu} \sum_{\sigma, \sigma \prime} c_{i \mu \sigma}^{\dagger} \sigma_{\sigma \sigma \prime}^{z} c_{i \mu \sigma \prime} \\
& \quad+\frac{\lambda}{2} \sum_{\eta} \sum_{i \mu \sigma, \nu \sigma \prime} c_{i \mu \sigma}^{\dagger} L_{\mu \nu}^{\eta} \sigma_{\sigma \sigma \prime}^{\eta} c_{i \nu \sigma \prime}+\mathcal{H}_{E},
\end{aligned}
$$

where we consider $d$ electrons, $c_{i \mu \sigma}^{\dagger}$ creates an electron at the $i$-th site with spin $\sigma$ and orbital index $\mu, t_{i j}^{\mu \nu}$ is the spin independent hopping between near neighbors, $J_{\mathrm{ex}}$ describes the spin splitting of up and down electrons in the ferromagnet, and $\lambda \vec{L} \cdot \vec{S}$ is the SOC term. In the TB model, the electric field induces asymmetry of the orbital lobes, which opens up new inter-orbital hopping channels, ${ }^{15-17}$ that were zero before. This is incorporated in the final term $\mathcal{H}_{E}$, having the same form as $\mathcal{H}_{\text {kin }}$, but with new matrix elements $t_{i j}^{\mu \nu}$, viz., $a=\left\langle x y\left|\mathcal{H}_{E}\right| y z\right\rangle_{\hat{x}}=\left\langle x y\left|\mathcal{H}_{E}\right| x z\right\rangle_{\hat{y}}, \beta=\left\langle x z\left|\mathcal{H}_{E}\right| z^{2}\right\rangle_{\hat{x}}$ $=\left\langle y z\left|\mathcal{H}_{E}\right| z^{2}\right\rangle_{\hat{y}}, \gamma=\left\langle x z\left|\mathcal{H}_{E}\right| x^{2}-y^{2}\right\rangle_{\hat{x}}=\left\langle x^{2}-y^{2}\left|\mathcal{H}_{E}\right| y z\right\rangle_{\hat{y}}$, which are roughly proportional to the electric field with the subscript $\hat{x}$ or $\hat{y}$ indicating the location of the nearest neighbor.

Te electric field breaks the inversion symmetry and leads to a Rashba interaction in the presence of SOC. The TB form $\mathcal{H}_{E}$ leads ${ }^{15,16}$ to the equivalent Rashba Hamiltonian in the momentum space ${ }^{18,19}$

$\mathcal{H}_{R}=a_{R}(\vec{\sigma} \times \vec{k}) \cdot \hat{z}$,

which results in the linear-k splitting of the band structure $\varepsilon_{k}=\frac{\hbar^{2} k^{2}}{2 m} \pm a_{R} k$, when $J_{\mathrm{ex}}=0$. The Rashba coefficients, which are

${ }^{1}$ Department of Physics \& Astronomy, University of Missouri, Columbia, MO 65211, USA

Correspondence: Sayantika Bhowal (bhowals@missouri.edu)

Received: 22 December 2018 Accepted: 5 May 2019

Published online: 21 May 2019 

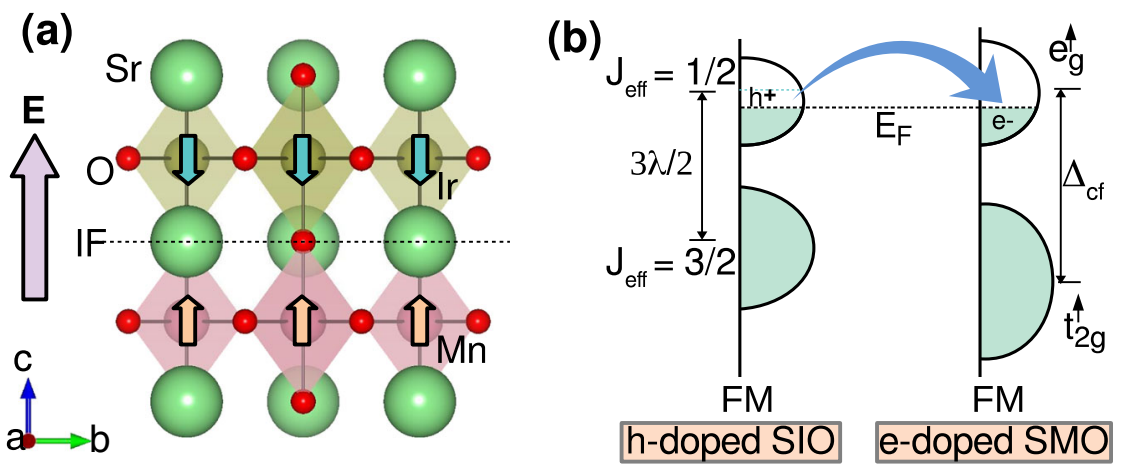

Fig. 1 Electronic and magnetic structure of the $(\mathrm{SIO})_{1} /(\mathrm{SMO})_{1}$ interface, both sides consisting of a single layer each, considered here as a specific example for the tuning of the AHC. The charge transfer across the interface leads to electron or hole doping, which in turn results in an emergent ferromagnetism at the interface, leading to an anomalous Hall effect
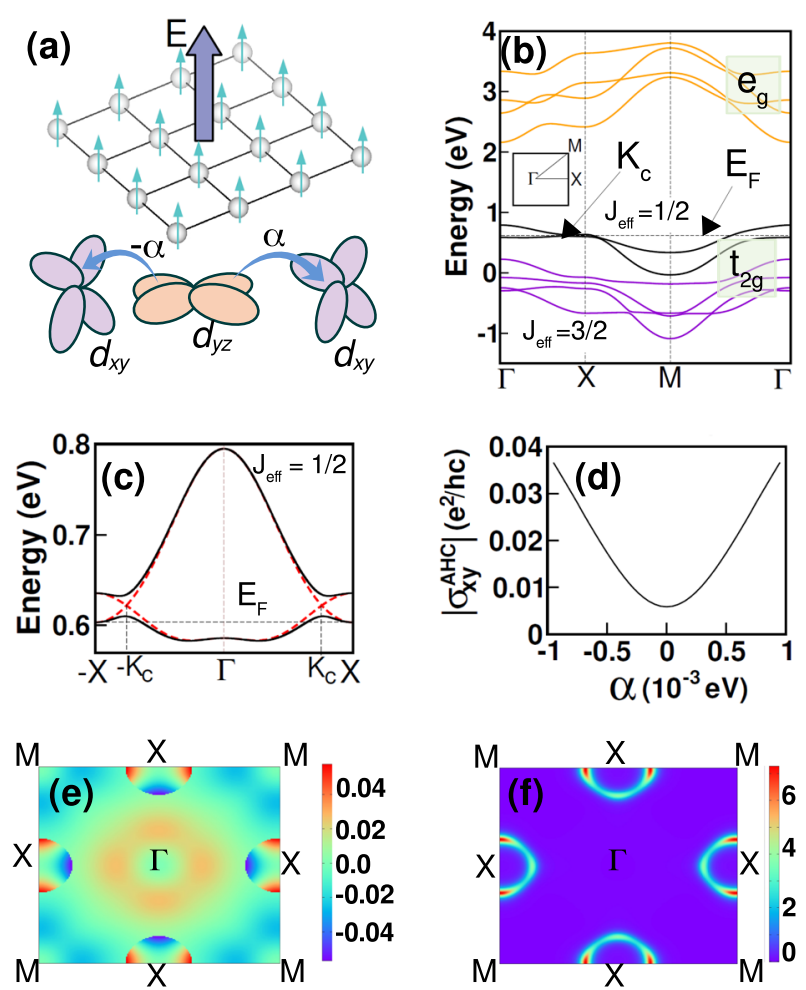

Fig. 2 Illustration of the electric field dependence of the Berry curvature and AHC, computed from Eqs. (2) and (3), for the squarelattice TB model. a The square lattice and the electric-field induced TB hopping integral $a$. b The TB band structure with both large crystal field $\Delta_{\text {cf }}$ and SOC parameter $\lambda$, which is relevant for SIO, where the $J_{\text {eff }}=1 / 2$ state is partially occupied. c Dispersion of the $J_{\text {eff }}$ $=1 / 2$ bands with and without an electric field (black and red lines, respectively). d Computed AHC for small electric fields, characterized by the parameter $a$, indicating the $\sigma_{x y}^{A H C} \propto|E|^{2}$ dependence. The Fermi energy $E_{F}$ corresponded to the electron concentration $n_{e}=0.9$ in the $J_{\text {eff }}=1 / 2$ bands. e, $\mathbf{f}$ Berry curvature $\Omega_{n}^{z}(\vec{k})$ (in units of $\AA^{2}$ ) for the lower $J_{\text {eff }}=1 / 2$ band without and with the electric field, respectively. $\Omega_{n}^{z}(\vec{k})$ is large near a crossing point $K_{c}$ (here close to $\mathrm{X}$ ) and has a dominant contribution to AHC. The TB parameters are: $V_{\sigma}$ $=-0.2 \mathrm{eV}(1 \mathrm{NN}),-0.1 \mathrm{eV}(2 \mathrm{NN}), V_{\sigma} / V_{\pi}=-1.85, J_{\mathrm{ex}}=0.5 \mathrm{eV}, \lambda=$ $0.4 \mathrm{eV}, a=\beta=\gamma=0.01 \mathrm{eV}(0$ if $E=0)$, and $\Delta_{\mathrm{cf}}=3 \mathrm{eV}$

different for different bands, depend on the strength of the SOC and can be expressed in terms of the matrix elements $\alpha, \beta$, and $\gamma$, all roughly proportional to $E$; For instance, in the present case with strong SOC, $a_{R} \approx 4 a / 3$ for the $J_{\text {eff }}=1 / 2$ states. In $3 D$ continuum, the SOC term $\mathcal{H}_{S O}=\frac{\hbar^{2}}{2 m^{2} c^{2}}(\vec{\nabla} V \times \vec{k}) \cdot \vec{\sigma}$, with the potential gradient $\vec{\nabla} V=-E \hat{z}$, immediately leads to the linear field dependence $a_{R}=\frac{\hbar^{2} E}{2 m^{2} c^{2}}$. In fact, the linear dependence is seen from the results of the full DFT calculations as shown in Fig. 4e. Note that Eq. (1) can be rewritten as $\mathcal{H}_{R}=\vec{B}_{k} \cdot \vec{\sigma}$, where $\vec{B}_{k}=a_{R}(\vec{k} \times \hat{z})$ can be thought of as a momentum dependent magnetic field, as stated in the Introduction, so that the Rashba interaction is anticipated to modify the AHC because an extra magnetic field has been introduced.

Another point to note here is that the origins of the SOC term $\lambda \vec{L} \cdot \vec{S}$ and the Rashba interaction are fundamentally the same, viz., the relativistic effect, where the electron sees an electric field (nuclear field or an applied field) as a magnetic field $\vec{B}=(\vec{v} \times \vec{E}) / c^{2}$ in its rest frame, which interacts with the spin of the electron. In fact, for a weak SOC, it can be shown that the Rashba coefficient $a_{R}$ is directly proportional to the strength of the SOC $\lambda \cdot{ }^{15,16}$ For the case of a strong SOC, the eigen states become spin-orbital entangled, and as a result $\left|a_{R}\right| \approx 4 a / 3$ for the $J_{\text {eff }}=1 / 2$ state, as stated above, is huge but independent of $\lambda .^{15,16}$

The AHC is computed ${ }^{20,21}$ from the momentum sum of the Berry curvature

$$
\sigma_{x y}^{\mathrm{AHC}}=-\frac{e^{2}}{\hbar} \frac{1}{N_{k} V_{c}} \sum_{n \vec{k}} \Omega_{n}^{z}(\vec{k}),
$$

where the sum is over the occupied states, and the Berry curvature $\Omega_{n}^{z}(\vec{k})$ for the $n$th band can be evaluated using the Kubo formula ${ }^{22}$

$$
\Omega_{n}^{z}(\vec{k})=-2 \hbar^{2} \sum_{n^{\prime} \neq n} \frac{\operatorname{Im}\left\langle\psi_{n \vec{k}}\left|v_{x}\right| \psi_{n^{\prime} \vec{k}}\right\rangle\left\langle\psi_{n^{\prime}}\left|v_{y}\right| \psi_{n \vec{k}}\right\rangle}{\left(\varepsilon_{n^{\prime} \vec{k}}-\varepsilon_{n \vec{k}}\right)^{2}} .
$$

Here $v_{\eta}=\hbar^{-1} \partial H / \partial k_{\eta}, V_{c}$ is the unit cell volume, and $N_{k}$ is the number of $k$ points used in the BZ sum. Near a band crossing point close to $E_{F}$, which we denote by $K_{c}$ [see Fig. $2 \mathrm{~b}, \mathrm{c}$ ], the denominator in (3) becomes small, leading to a large contribution to the AHC. For a crossing point deep below $E_{F}$, the contributions to the $A H C$ from the two crossing bands cancel due to the opposite signs of the matrix elements.

The computed values of the Berry curvature using these expressions for the TB model in absence and presence of electric field are shown in Fig. 2e, $f$ respectively, from which it is clear that the band crossing points have the dominant contributions to the Berry curvature. The calculated AHC for small electric fields, characterized by the field-induced TB parameter $a$ in $\mathcal{H}_{E}$, is shown in Fig. 2d, which indicates the square-law dependence $\sigma_{x y}^{\mathrm{AHC}}=\sigma_{0}+c E^{2}$. The AHC can also be tuned by doping, which adds carriers to the system. The results obtained for the TB model are summarized in Fig. 3, indicating the strong dependence of the $\mathrm{AHC}$ on the applied electric field, characterized by the parameter $a$, as well as the electron concentration $n_{e}$, which can be modified by doping. Note that our TB model addresses the electric field 


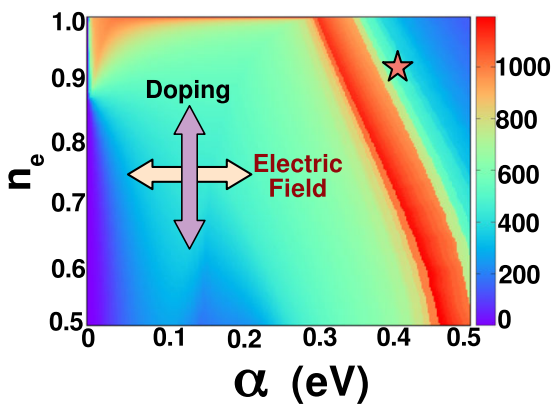

Fig. 3 Variation of the AHC with electric field, parametrized by $a$, and the carrier concentration $n_{e}$ [electrons in the $J_{\text {eff }}=1 / 2$ band; see Fig. 2c], computed for the TB model. The star corresponds to parameters for SIO/SMO and units of AHC are $\Omega^{-1} \mathrm{~cm}^{-1}$

dependence of the AHC using a general ferromagnetic square lattice. It is not a model for the bilayer structure considered in our DFT calculations, and therefore does not reproduce the specific band structure.

The fact that $\sigma_{x y}^{\mathrm{AHC}} \propto|E|^{2}$ for small electric fields can be understood by considering a two-band model near the crossing point $\vec{K}_{c}$

$$
\mathcal{H}(\vec{q})=\left[\begin{array}{cc}
\eta q & h_{12} \\
h_{12}^{*} & -\eta q
\end{array}\right],
$$

where for $E=0$, we have the conical bands $\varepsilon_{ \pm}= \pm \eta q$, and $h_{12}$ is the electric field dependent term. Explicitly, we take the crossing point in the $J_{\text {eff }}=1 / 2$ band, so that the TB form of $\mathcal{H}_{E}$ yields the expression $h_{12}=a_{R}\left(\sin k_{y}+i \sin k_{x}\right)$, where $a_{R}=4 a / 3$, obtained straightforwardly from the Bloch functions corresponding to the $J_{\text {eff }}=1 / 2$ wave functions: $\psi_{ \pm}=(|y z, \bar{\sigma}\rangle \pm i|x z, \bar{\sigma}\rangle \pm|x y, \sigma\rangle) / \sqrt{3}$. From the eigenvalues of Eq. (4), viz., $\varepsilon_{ \pm}= \pm \sqrt{\eta^{2} q^{2}+\left|h_{12}\right|^{2}}$, and the corresponding wave functions, we find the Berry curvature from Eq. (3) to be

$$
\Omega_{ \pm}^{z}= \pm \frac{\eta}{2} \times \frac{-a_{1} a_{2} q+c_{1} \sin \theta-c_{2} \cos \theta-a_{1} a_{2} q \cos (2 \theta)}{\left(\eta^{2} q^{2}+\left|h_{12}\right|^{2}\right)^{3 / 2}}
$$

where we have kept the terms to the lowest order in $\vec{q} \equiv \vec{k}-\vec{K}_{c^{\prime}}$ so that $h_{12}=\Delta+a_{1} q_{y}+i a_{2} q_{x}$, where $a_{1}=a_{R} \cos K_{y}, a_{2}=a_{R} \cos K_{x}$, and $\Delta=a_{R}\left(\sin K_{y}+i \sin K_{x}\right)$. In Eq. (5), $c_{1}=a_{2} \operatorname{Re}(\Delta), c_{2}=a_{1} \operatorname{Im}(\Delta)$, and the \pm sign refers to the upper and the lower bands. For $a \ll \eta$, valid for small electric fields, we immediately find the angleintegrated Berry curvature to be

$I_{ \pm}(q) \equiv \int_{0}^{2 \pi} \Omega_{ \pm}^{z}(q, \theta) d \theta=\mp \frac{f a_{R}^{2} q \eta}{2\left(\eta^{2} q^{2}+|\Delta|^{2}\right)^{3 / 2}}$,

where $f=\cos K_{x} \times \cos K_{y}$. This equation together with Eq. (2) clearly shows that $\sigma_{x y}^{A H C} \propto|E|^{2}$, since the Rashba coefficient $a_{R}$ scales as the electric field strength. Furthermore, it is clear that $I_{ \pm}(q)$ is sharply peaked close to the band crossing point. In the squarelattice model, we find the AHC to scale as: $\sigma_{x y}^{\mathrm{AHC}}=\sigma_{0}+c E^{2}$ [see Fig. 2d] for very small electric field, where $\sigma_{0} \neq 0$ due to the broken time-reversal symmetry, which is present even with $E=0$. Note that this result is valid only for small $E$. For sufficiently large $E$, the bands can realign which can shift the Fermi level, and the pre-factor $c$ can get modified as well, sometimes even becoming negative, as seen from the DFT results (Fig. 5) for a large positive electric field. This is further elaborated in the Supplementary Information.

We now turn to the DFT calculations for the (001) $(\mathrm{SIO})_{1} /(\mathrm{SMO})_{1}$ slab to illustrate the field tuning effect for a real material. We used the plane wave methods to solve the DFT equations within the $\mathrm{GGA}+\mathrm{SOC}+\mathrm{U}$ approximation. ${ }^{23-25}$ The AHC was calculated by computing the Berry curvature using the Wannier interpolation
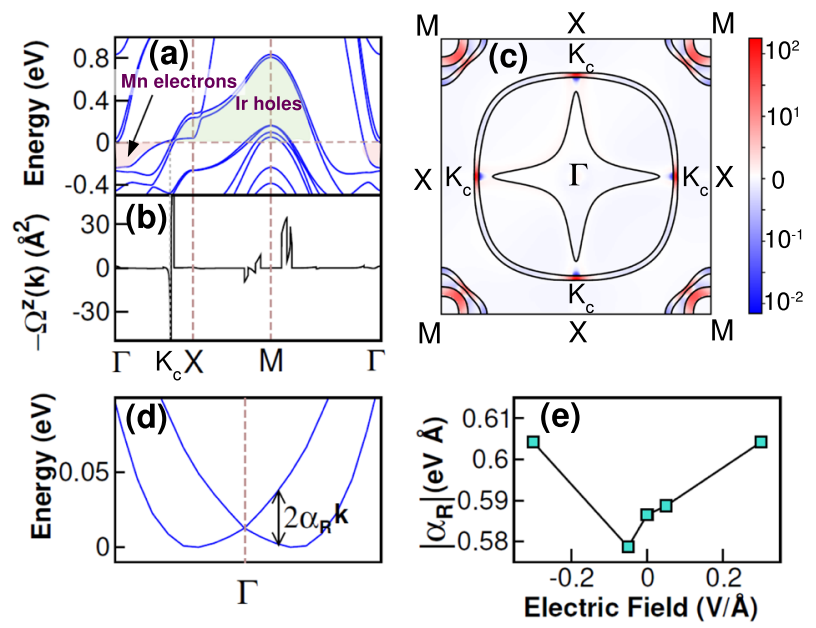

Fig. 4 DFT results for the $\mathrm{AHC}$ of the $(\mathrm{SIO})_{1} /(\mathrm{SMO})_{1}$ slab with an applied electric field $\mathrm{E}$. a Band structure for $\mathrm{E}=-0.3 \mathrm{~V} / \AA$, with the colored regions indicating the Ir $\mathrm{t}_{2 g}$ holes and $M n \mathrm{e}_{g}$ electrons, and the band crossing point $K_{c}$. b The Berry curvature $\Omega_{n}^{z}(\vec{k})$ summed over the occupied bands $n$ at each $\vec{k}$ point along the specified line in the BZ. c Contours of the same quantity in (b) in the $k_{x}-k_{y}$ plane, indicating the large contributions from regions around $M$ and $K_{c}$ points in the $B Z$, with the latter providing the dominant contribution to the field dependence of the AHC as discussed in the text. $\mathbf{d}$ Rashba splitting of the DFT band structure for the non-magnetic state, used for extracting $a_{R}$. e Variation of the computed $a_{R}$ with the applied electric field

approach as implemented in the Wannier90 code. $^{26}$ Further details are given in the Supplementary Information.

A key feature of the electronic structure of the (001) $(\mathrm{SIO})_{1} /$ $(\mathrm{SMO})_{1}$ interface is the charge transfer ${ }^{14,27}$ from the spin-orbital entangled $J_{\text {eff }}=1 / 2$ state on the SIO side to the empty $M n-e_{g}$ states on the SMO side [Fig. 1b]. The charge transfer is important because it helps drive ferromagnetism at the interface, thereby breaking the time-reversal symmetry, which is an essential ingredient for AHC. The electron-doped SMO becomes ferromagnetic due to the Anderson-Hasegawa-DeGennes double exchange, ${ }^{28,29}$ as is well known from the manganite physics, while SIO becomes ferromagnetic due to a combination of the magnetic proximity effect and hole doping. The magnetic proximity effect arising from the presence of the neighboring ferromagnetic SMO layer is due to the exchange interaction across the interface, while the hole doping, in addition, has a tendency to drive the SIO part ferromagnetic due to the Nagaoka physics, where in the infinite-U limit, a single doped carrier in the half-filled Hubbard model destroys the anti-ferromagnetic insulating ground state, driving the system into a ferromagnetic metal. ${ }^{30}$ In fact, a short range ferromagnetic interaction has been observed experimentally in the hole-doped $\mathrm{SIO}^{31}$ Both our DFT calculation as well as X-ray magnetic circular dichroism measurement ${ }^{9}$ show that the $\mathrm{Ir}$ and $\mathrm{Mn}$ spins are antiparallel to each other as indicated in Fig. 1a.

For the (001) $(\mathrm{SIO})_{1} /(\mathrm{SMO})_{1}$ slab, we find that there is a transfer of about $0.08|e|$ across the interface, enough to make the SMO side ferromagnetic via double exchange. The charge transfer is consistent with the fraction of the area in the Brillouin zone occupied by the $M n\left(e_{g}\right)$ states as indicated by the purple region in Fig. 4a. Such a charge transfer has indeed been observed in the experiments. However, the magnitude of the observed charge transfer $0.5|e|$ is significantly higher, which may be attributed to the fact that charge partitioning in the solid is an ill-defined quantity because creation of disjoint volumes associated with ions is not unique. Note that, the experiments were performed for the 
superlattice structure as opposed to the $(\mathrm{SIO})_{1} /(\mathrm{SMO})_{1}$ slab considered here.

The magnetic structure is in good agreement with the measured value for the superlattice. We find the spin (orbital) moment to be $3.12 \mu_{B}\left(0.03 \mu_{B}\right)$ for $\mathrm{Mn}$, while for Ir, it is $0.14 \mu_{B}$ $\left(0.08 \mu_{B}\right)$, which are similar to the values for the superlattice structure. Total energy calculations with constrained spin directions indicate the moments to be aligned along $\hat{z}$ (normal to the plane) in agreement with the experimental results as well. ${ }^{9}$ In order to test the results from the DFT calculations, we first computed the AHC for the (001) $(\mathrm{SIO})_{1} /(\mathrm{SMO})_{1}$ superlattice structure with $E=0$, for which the $\mathrm{AHC}$ has been measured. The computed value $\sigma_{x y}^{\text {AHC }} \approx 26 \Omega^{-1} \mathrm{~cm}^{-1}$ is in reasonable agreement with the experimental value of $\sim 18 \Omega^{-1} \mathrm{~cm}^{-1}$.

The typical band structure for the $(\mathrm{SIO})_{1} /(\mathrm{SMO})_{1}$ is shown in Fig. $4 a$, where the Ir holes and the Mn electrons are shown, which is consistent with the charge transfer across the interface, as sketched in Fig. 1. It is essential to optimize the crystal structure for each case in order to take into account the electrostatic screening effect, which reduces the applied field. The only changes in the band structure occur around $\vec{K}_{c}$, for different electric fields, but the overall band structure remains the same, and there is no substantial change of the charge transfer up to the electric fields we used in the calculations.

As already mentioned, large contributions to the AHC comes from regions in the $B Z$, where both occupied and unoccupied bands occur near the Fermi energy for same $\vec{k}$, which can be seen from the small energy denominator in the Kubo formula (3). As seen from Figs. $4 \mathrm{~b}$ and 5 , there are two regions with significant contributions to the $\mathrm{AHC}, \sigma_{c}$ from the region around the four crossing points $K_{c}$, which strongly varies with the electric field, and the remaining part $\sigma_{\text {rest }}$, which remains more or less unaffected because unlike near $K_{c}$, the bands change very little at $M$, which is the major contributor to $\sigma_{\text {rest }}$ (see the Fig. 3 of the Supplementary Information for the evolution of the band structure with $E$ ). The electric field is expected to modify the Rashba coefficient as $a_{R}=$ $\frac{\hbar^{2} E}{2 m^{2} c^{2}}$ in the free particle model as mentioned already. To evaluate this for the solid, we computed the Rashba coefficient $a_{R}$ as a function of the electric field from the linear band splitting $\Delta_{k}=$ $2 a_{R} k$ near the $\Gamma$ point from additional DFT calculations for the nonmagnetic structure. The results, Fig. $4 d$, e, show the anticipated linear $E$ dependence of $a_{R}$. Note that for $E=0, a_{R}$ is large, which can be attributed to an intrinsic electric field $E_{0}$ that exists at the interface due to the broken inversion symmetry.

The computed $\sigma_{x y}^{\mathrm{AHC}}$, presented in Fig. 5, shows a strong electric field dependence. Even though the band structure of the slab is

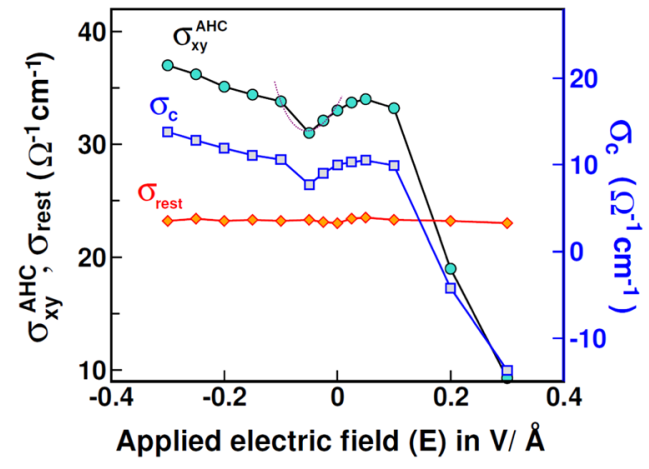

Fig. 5 The variation of total $\mathrm{AHC}\left(\sigma_{\mathrm{xy}}^{\mathrm{AHC}}=\sigma_{\mathrm{c}}+\sigma_{\text {rest }}\right)$, and the partial contributions, $\sigma_{c}$ from the crossing point $K_{c}$ in the BZ, and the remaining part $\sigma_{\text {rest }}$, as a function of the applied electric field $E$. The dashed parabola indicates the remnant of the square electric field dependence for the small electric field. It is clear that $\sigma_{\text {rest }}$ shows little change with $E$, while $\sigma_{c}$ changes significantly, controlling the electric field behavior of the AHC much more complex than the single band crossing in the model, still the remnant of a square electric-field-dependence is seen for small electric fields. This is indicated by the dashed parabola in Fig. 5 , with the relation $\sigma_{x y}^{\mathrm{AHC}} \approx \sigma_{0}+c\left(E+E_{0}\right)^{2}$, where $E_{0} \approx 0.05 \mathrm{~V} / \AA$ is the intrinsic electric field at the interface and $E$ is the applied electric field. For large positive electric fields, the band structure changes a lot, resulting in a large change in the $A H C$, as discussed in the Supplementary Information. Thus the tuning of AHC is achievable in the real material over a range of electric field as evident from Fig. 5. We note that calculations with a larger value of the Coulomb $\mathrm{U}(\mathrm{U}=4 \mathrm{eV}$ for $\mathrm{Mn}$ and $\mathrm{U}=3 \mathrm{eV}$ for Ir) yields a larger $\mathrm{AHC}\left(55.3,57,57.9 \Omega^{-1} \mathrm{~cm}^{-1}\right.$ for $E=-0.05,0$, and $0.05 \mathrm{~V} / \AA$, respectively); however, the trend obtained for the electric field dependence remains intact.

Note that we used the $\Gamma$ point to evaluate the strength of the Rashba interaction because of the characteristic linear band splitting, which allows for a convenient evaluation of $a_{R}$ there. At other points in the Brillouin zone, such as X or M, the Rashba coefficient will be proportional. This point can be argued by considering the expression for the relativistic magnetic field $(\vec{v} \times \vec{E}) / c^{2}$ seen by the electron in its rest frame, which leads to the Rashba interaction (1) for a parabolic band $\varepsilon_{k}=\hbar^{2} k^{2} / 2 m$. For a general $k$ point in the Brillouin zone, $\vec{v}=\frac{1}{\hbar} \vec{\nabla}_{k} \varepsilon_{k}$ which leads to the form $H_{R}=a_{R}^{\prime}(\vec{\sigma} \times \vec{v}) \cdot \hat{z}$, where $a_{R}^{\prime}$ is proportional to $a_{R}$.

So far, we described the electric field tuning via the modification of the Rashba SOC by the applied electric field. A second way to alter the AHC is by manipulating the carrier density by doping. This is verified by shifting the Fermi energy in the DFT calculations to a lower value, thereby increasing the Ir-hole concentration. In presence of an electric field $E$, shifting of Fermi energy downwards by $\Delta \varepsilon_{F}=-0.1 \mathrm{eV}$ enhances the AHC by $15 \%$ to about $38 \Omega^{-1}$ $\mathrm{cm}^{-1}$. For $\Delta \varepsilon_{F}=-0.15 \mathrm{eV}$, it is further increased to the value $85 \Omega^{-1} \mathrm{~cm}^{-1}$. This offers an additional tool for the manipulation of the AHC.

In conclusion, we have shown that the anomalous Hall effect at the $3 d-5 d$ interfaces can be tuned by modifying the Rashba spinorbit interaction with the application of an external electric field. The major contribution to the electric-field dependence comes from the band-crossing points close to the Fermi energy. In addition, the AHC can be tuned by manipulating the electron density with doping. We illustrated the results with a ferromagnetic square-lattice model as well as with density-functional calculations for the recently grown manganite-iridate interface, viz., (001) $(\mathrm{SIO})_{1} /(\mathrm{SMO})_{1}$. In fact, several recent experiments 32,33 have found evidence for the electric field dependence of the AHC in the oxide heterostructures. It would be valuable to develop this effect further, both theoretically and experimentally, with an eye towards potential spintronics applications.

\section{METHODS}

The magnetic properties of SIO/SMO are studied using DFT calculations based on the plane-wave based projector augmented wave (PAW) ${ }^{34,35}$ method as implemented in the Vienna ab initio simulation package $(V A S P)^{23}$ within the generalized gradient approximation (GGA) ${ }^{25}$ including Hubbard $U^{36}$ and SOC. The kinetic energy cut-off of the plane wave basis was chosen to be $550 \mathrm{eV}$. Following the previous report, ${ }^{14}$ all the calculations have been performed using $\mathrm{U}=2 \mathrm{eV}$ for $\mathrm{Ir}$ and $\mathrm{U}=3 \mathrm{eV}$ for $\mathrm{Mn}-d$ states respectively unless stated otherwise. In order to take into account the electrostatic screening effects, we have relaxed the structure in presence of each of the electric fields using VASP until the HellmanFeynman forces on each atom becomes less than $0.01 \mathrm{eV} / \AA$. For the calculations in presence of electric field, a sawtooth-like potential is applied.

The AHC of the slabs in presence and absence of electric field are calculated using QUANTUM ESPRESSO and the Wannier interpolation approach. $^{24,26}$ In order to compute the AHC, BZ integration of the Berry curvature is performed with a k-mesh of $400 \times 400 \times 80$, and in the region where the Berry curvature is sharply peaked (as indicated by a Berry curvature sum over the occupied states being larger than $100 \AA^{2}$ ), an 
"adaptively refined" mesh ${ }^{26}$ of $7 \times 7 \times 7$ is used. The convergence is confirmed by using finer mesh points.

\section{DATA AVAILABILITY}

All data generated and/or analyzed during this study are included in this article and its Supplementary Information file.

\section{ACKNOWLEDGEMENTS}

We thank the U.S. Department of Energy, Office of Basic Energy Sciences, Division of Materials Sciences and Engineering for financial support under Grant No. DEFG02O0ER45818.

\section{AUTHOR CONTRIBUTIONS}

S.B. performed the model and the DFT calculations. Both the authors developed the concepts, contributed to the discussions of the results, and the writing of the manuscript.

\section{ADDITIONAL INFORMATION}

Supplementary Information accompanies the paper on the npj Computationa Materials website (https://doi.org/10.1038/s41524-019-0198-8).

Competing interests: The authors declare no competing interests.

Publisher's note: Springer Nature remains neutral with regard to jurisdictional claims in published maps and institutional affiliations.

\section{REFERENCES}

1. Hall, E. H. On a new action of the magnet on electric currents. Am. J. Math. 2, 287 (1879).

2. Hall, E. H. On the new action of magnetism on a permanent electric current Philos. Mag. 10, 301 (1880).

3. Karplus, R. \& Luttinger, J. M. Hall effect in ferromagnetics. Phys. Rev. 95, 1154 (1954).

4. Gerber, A. et al. Extraordinary Hall effect in magnetic films. J. Magn. Magn. Mater. 242, 90 (2002)

5. Takeda, T. \& Ohara, S. Magnetic structure of the cubic perovskite Type $\mathrm{SrMnO}_{3}$. J. Phys. Soc. Jpn. 37, 275 (1974).

6. Zhao, J. G. et al. High-pressure synthesis of orthorhombic $\mathrm{SrlrO}_{3}$ perovskite and its positive magnetoresistance. J. Appl. Phys. 103, 103706 (2008).

7. Zeb, M. A. \& Kee, H.-Y. Interplay between spin-orbit coupling and Hubbard interaction in $\mathrm{SrlrO}_{3}$ and related Pbnm perovskite oxides. Phys. Rev. B 86, 085149 (2012).

8. Zheng, $\mathrm{H}$. et al. Simultaneous metal-insulator and antiferromagnetic transitions in orthorhombic perovskite iridate $\mathrm{Sr}_{0.94} \mathrm{Ir}_{0.78} \mathrm{O}_{2.68}$ single crystals. Phys. Rev. B 93 235157 (2016).

9. Nichols, J. et al. Emerging magnetism and anomalous Hall effect in iridatemanganite heterostructures. Nat. Commun. 7, 12721 (2016).

10. Matsuno, J. et al. Engineering a spin-orbital magnetic insulator by tailoring superlattices. Phys. Rev. Lett. 114, 247209 (2015).

11. Fan, W. \& Yunoki, S. Electronic and magnetic structure under lattice distortion in $\mathrm{SrlrO}_{3} / \mathrm{SrTiO}_{3}$ superlattice: a first-principles study. J. Phys.: Conf. Ser. 592, 012139 (2015).

12. Pang, B. et al. spin-glass-like behavior and topological Hall effect in $\mathrm{SrRuO}_{3} / \mathrm{SrlrO}_{3}$ superlattices for oxide spintronics applications. ACS Appl. Mater. Interfaces 9, 3201-3207 (2017).

13. Kim, J.-W. et al. Controlling entangled spin-orbit coupling of $5 \mathrm{~d}$ states with interfacial heterostructure engineering. Phys. Rev. B 97, 094426 (2018).

14. Okamoto, S. et al. Charge transfer in iridate-manganite superlattices. Nano Lett. 17, 2126-2130 (2017).
15. Shanavas, K. V. \& Satpathy, S. Electric field tuning of the rashba effect in the polar perovskite structures. Phys. Rev. Lett. 112, 086802 (2014).

16. Shanavas, K. V., Popović, Z. S. \& Satpathy, S. Theoretical model for Rashba spinorbit interaction in d electrons. Phys. Rev. B 90, 165108 (2014).

17. Petersen, L. \& Hedegard, P. A simple tight-binding model of spin-orbit splitting of sp-derived surface states. Surf. Sci. 459, 49 (2000).

18. Rashba, E. I. Properties of semiconductors with an extremum loop. 1. Cyclotron and combinational resonance in a magnetic field perpendicular to the plane of the loop. Sov. Phys. Solid State 2, 1109 (1960).

19. Bychkov, Y. A. \& Rashba, E. I. Oscillatory effects and the magnetic susceptibility of carriers in inversion layers. J. Phys. C. 17, 6039 (1984).

20. Chang, M.-C. \& Niu, Q. Berry phase, hyperorbits, and the Hofstadter spectrum Phys. Rev. Lett. 75, 1348 (1995).

21. Chang, M.-C. \& Niu, Q. Berry phase, hyperorbits, and the Hofstadter spectrum: semiclassical dynamics in magnetic Bloch bands. Phys. Rev. B. 53, 7010 (1996).

22. Thouless, D. J., Kohmoto, M., Nightingale, M. P. \& den Nijs, M. Quantized Hall conductance in a two-dimensional periodic potential. Phys. Rev. Lett. 49, 405 (1982).

23. Kresse, G. \& Furthmüller, J. Efficient iterative schemes for ab initio total-energy calculations using a plane-wave basis set. Phys. Rev. B 54, 11169 (1996).

24. Giannozzi, P. et al. QUANTUM ESPRESSO: a modular and open-source software project for quantum simulations of materials. J. Phys. Condens. Matter 21, 395502 (2009).

25. Perdew, J. P., Burke, K. \& Ernzerhof, M. Generalized gradient approximation made simple. Phys. Rev. Lett. 77, 3865 (1996).

26. Wang, X., Yates, J. R., Souza, I. \& Vanderbilt, D. Ab initio calculation of the anomalous Hall conductivity by Wannier interpolation. Phys. Rev. B 74, 195118 (2006).

27. Bhowal, S. \& Satpathy, S. Emergent magnetism at the $3 d-5 d$ interface: $\mathrm{SrMnO}_{3} /$ $\mathrm{SrlrO}_{3}$. AlP Conf. Proc. 2005, 020007 (2018).

28. Anderson, P. W. \& Hasegawa, H. Considerations on double exchange. Phys. Rev. 100, 675 (1955).

29. DeGennes, P.-G. Effects of double exchange in magnetic crystals. Phys. Rev. 118, 141 (1960)

30. Nagaoka, Y. Ferromagnetism in a narrow, almost half-filled $s$ band. Phys. Rev. 147, 392 (1966).

31. Qasim, I., Kennedy, B. J. \& Avdeev, M. Stabilising the orthorhombic perovskite structure in SrlrO3 through chemical doping. Synthesis, structure and magnetic properties of $\mathrm{Srlr}_{1-x} \mathrm{Mg}_{x} \mathrm{O}_{3}(0.20 \leq \mathrm{x} \leq 0.33)$. J. Mater. Chem. A 1, 13357 (2013).

32. Ohuchi, Y. et al. Electric-field control of anomalous and topological Hall effects in oxide bilayer thin films. Nat. Commun. 9, 213 (2018).

33. Mizuno, H. et al. Electric-field-induced modulation of the anomalous Hall effect in a heterostructured itinerant ferromagnet $\mathrm{SrRuO}_{3}$. Phys. Rev. B 96, 214422 (2017).

34. Blochl, P. E. Projector augmented-wave method. Phys. Rev. B 50, 17953 (1994).

35. Kresse, G. \& Joubert, D. From ultrasoft pseudopotentials to the projector augmented-wave method. Phys. Rev. B 59, 1758 (1999).

36. Anisimov, V. I., Zaanen, J. \& Andersen, O. K. Band theory and Mott insulators: Hubbard U instead of Stoner I. Phys. Rev. B 44, 943 (1991).

(2) Open Access This article is licensed under a Creative Commons adaptation, distribution and reproduction in any medium or format, as long as you give appropriate credit to the original author(s) and the source, provide a link to the Creative Commons license, and indicate if changes were made. The images or other third party material in this article are included in the article's Creative Commons license, unless indicated otherwise in a credit line to the material. If material is not included in the article's Creative Commons license and your intended use is not permitted by statutory regulation or exceeds the permitted use, you will need to obtain permission directly from the copyright holder. To view a copy of this license, visit http://creativecommons org/licenses/by/4.0/.

(c) The Author(s) 2019 